models. AMG 570 binding affinity to human and cyno ICOSL/BAFF was tested by Kinexa A. An ongoing, first-inhuman study has enrolled healthy adult subjects into 6 escalating single-dose cohorts. Eight participants were enrolled into each cohort and randomized 3:1 to receive AMG 570 or placebo. The primary endpoint was treatment-emergent adverse events (AEs). Secondary endpoints included pharmacokinetics and pharmacodynamics.

Results ICOSL and BAFF dual inhibition was more effective than single inhibition in ameliorating arthritis incidence and severity in the mouse CIA model as well as reducing antidsDNA IgG, delaying proteinuria and improving survival in the NZB/NZW lupus model. Based on high affinity to ICOSL and BAFF, AMG 570 was selected for investigation in a single ascending dose study in healthy subjects. As of an ad hoc interim analysis following six cohorts, 48 healthy participants received one dose of investigational product (AMG 570 or placebo). Overall, 73 mild to moderate AEs were reported. The most common AEs were upper respiratory tract infection and injection site erythema. No drug-related serious AEs or fatal AEs were reported thus far. AMG 570 demonstrated nonlinear pharmacokinetics consistent with cell surface ICOSL binding. At the highest dose tested, AMG 570 achieved $>90 \%$ mean ICOSL receptor occupancy on circulating $\mathrm{B}$ cells 8 days after dosing. AMG 570 led to a reduction in circulating naïve $B$ cells and an increase in circulating memory B cells.

Conclusions Dual inhibition of ICOSL and BAFF is more efficacious than single target inhibition in mouse disease models. In healthy subjects to date, single doses of AMG 570 have been safe, well tolerated, and demonstrated pharmacodynamic activity consistent with inhibition of both ICOSL and BAFF. Funding Source(s): Amgen Inc.

\section{ASSESSMENT OF THE QRISK2, QRISK3, SLE CARDIOVASCULAR RISK EQUATION, MODIFIED FRAMINGHAM AND FRAMINGHAM RISK CALCULATORS AS PREDICTORS OF CARDIOVASCULAR DISEASE EVENTS IN SYSTEMIC LUPUS ERYTHEMATOSUS}

${ }^{1}$ Jagan Sivakumaran*, ${ }^{2}$ Paula Harvey, ${ }^{1}$ Ahmed Omar, ${ }^{3}$ Murray B Urowitz, ${ }^{3}$ Dafna D Gladman, ${ }^{4}$ Nicole Anderson, ${ }^{4}$ Jiandong Su, ${ }^{3}$ Zahi Touma. ${ }^{1}$ University of Toronto; ${ }^{2}$ Division of Cardiology, Women's College Hospital; ${ }^{3}$ Krembil Research Institute, University of Toronto; ${ }^{4}$ The Centre for Prognosis Studies in the Rheumatic Diseases Toronto Western Hospital

\subsection{6/lupus-2019-Ism.291}

Background Systemic lupus erythematosus (SLE) is recognised as an independent risk factor for cardiovascular disease (CVD). This study aimed to determine which cardiovascular risk assessment tool: QRISK2, QRISK3, Framingham (FRS), Modified FRS (mFRS) or SLE Cardiovascular Risk Equation (SLECRE) best predicts CVD in SLE. QRISK3, mFRS and SLECRE are CVD risk assessment instruments considering SLE in risk prognosticating patients.

Methods Single-centre analyses on prospectively collected data of 1887 SLE patients were performed to compute 10 year CVD risk scores for each tool. Tools scores were evaluated against CVD development at or within ten years for cases (CVD events) and controls (no CVD events). For cases, the index date for risk score calculation was chosen 10 years, or as close to 10 years as possible prior to the CVD event. Similarly, for controls, risk scores were calculated as close to 10 years as possible prior to the most recent clinic appointment. Proportions of patients classified as low risk $(<10 \%)$, median risk $(10 \%-20 \%)$ and high risk $(>20 \%)$ of developing CVD according to each tool were determined. Sensitivity, specificity, positive/negative predictive values and c-statistics of these tools were analysed.

Results 232 total CVD events were seen in the cohort including myocardial infarction, stroke, transient ischemic attack, heart failure and CVD death. QRISK2 and FRS performances were similar, while the QRISK3 and mFRS performances were similar. The SLECRE classified the highest number of patients

Abstract 291 Table 1 Percentage of cases and controls classified as low $(<10 \%)$, median (10-20\%) and high risk of developing CVD according to each CVD risk assessment tool

\begin{tabular}{|c|c|c|c|c|}
\hline Tool & CVD Status & $\begin{array}{c}\text { Low Risk } \\
(<\mathbf{1 0} \mathbf{( \% )})\end{array}$ & $\begin{array}{c}\text { Median Risk } \\
(\mathbf{1 0}-\mathbf{2 0} \mathbf{)}) \mathbf{( \% )}\end{array}$ & $\begin{array}{c}\text { High Risk } \\
(\mathbf{> 2 0} \mathbf{)}) \mathbf{( \% )}\end{array}$ \\
\hline \multirow{2}{*}{ QRISK2 } & Cases & 81 & 14 & 4 \\
\hline \multirow{2}{*}{ FRS } & Controls & 93 & 5 & 2 \\
\hline \multirow{2}{*}{ QRISK3 } & Cases & 78 & 16 & 6 \\
\hline \multirow{2}{*}{ mFRS } & Controls & 93 & 6 & 1 \\
\hline & Cases & 53 & 27 & 20 \\
\hline \multirow{2}{*}{ SLECRE } & Controls & 78 & 13 & 9 \\
\hline & Cases & 54 & 25 & 21 \\
\hline & Controls & 83 & 10 & 7 \\
\hline
\end{tabular}


as median-high risk (table 1). The sensitivities and specificities are as follows for each tool: QRISK2 (19\%, 93\%), FRS (22\%, 93\%), mFRS (46\%, 83\%), QRISK3 (47\%, 78\%), SLECRE $(61 \%, 63 \%)$, respectively. The tools were similar in negative predictive value, ranging from $89 \%$ (QRISK2) to $92 \%$ (SLECRE). The FRS and mFRS had the greatest c-statistics, both equalling 0.73 , demonstrating the greatest predictive accuracy amongst the tools, while the QRISK3 and SLECRE had the lowest (0.67).

Conclusions While the mFRS performance was superior to the FRS, the QRISK3 did not outperform the mFRS. Although the SLECRE had the highest sensitivity, it had the lowest specificity, demonstrated by grouping the most cases and controls in the median-high risk category. Several factors are important to consider when deciding which risk assessment tools to utilize clinically: ease of use, sensitivity/specificity, and laboratory data accessibility. Thus, the mFRS continues to be a practical tool with a simple, intuitive scoring system appropriate for the ambulatory clinic setting based on the initial weighting of the FRS while adjusting for SLE.

Funding Source(s): Lupus Foundation of America - Gina M. Finzi Memorial Student Summer Fellowship

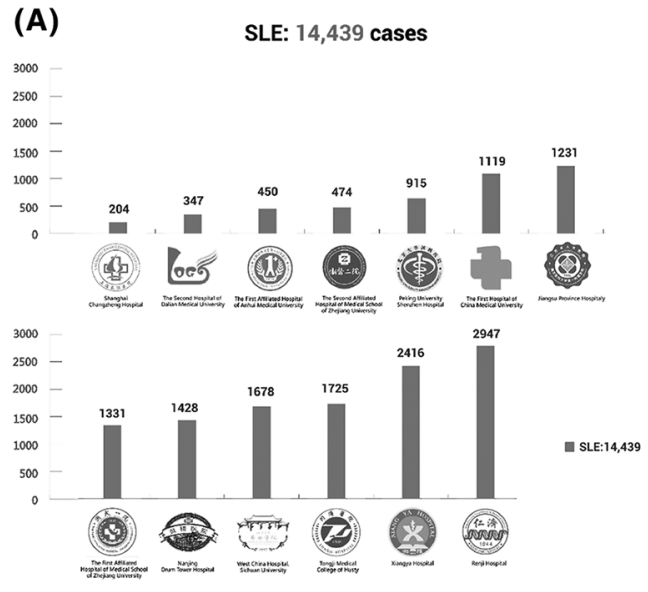

(C)

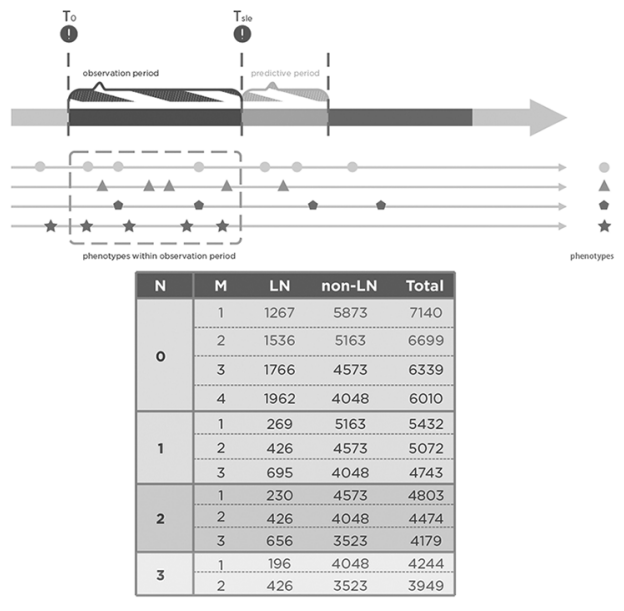

Cardiovascular Disease Risk Stratification by Risk Assessment Tool

\section{ARTIFICIAL INTELLIGENCE PREDICT THE LUPUS NEPHRITIS BASED ON FULL-PHENOTYPE DATABASE WITH NATURAL LANGUAGE PROCESSING TECHNOLOGY}

${ }^{1}$ Ting Li*, 2 Lei Jiang, ${ }^{3}$ Li Wang, ${ }^{4}$ Chunde Bao, ${ }^{5} \mathrm{Huji}$ Xu. 'Shanghai Jiaotong University School of Medicine affiliated Renji Hospital; ${ }^{2}$ Department of Rheumatology and Immunology, Changzheng Hospital, Second Military Medical UniversityShanghai200433China; ${ }^{3}$ Department of medical informatics, Medical School, Nantong University, 19 Qixiu Road, 226001 Nantong, Jiangsu Province, China; ${ }^{4}$ Renji Hospital, School of Medicine, Shanghai Jiao Tong University, China; ${ }^{5}$ The Second Military Medical University

\subsection{6/lupus-2019-Ism.292}

Background As the prototype of autoimmune disease, systemic lupus erythematosus (SLE) has complex and diverse clinical manifestations which may be harmful even life-threaten. Its pitiful that we can just passively respond to these serious complications. It will be a great advantage if the high-risk groups

(B)
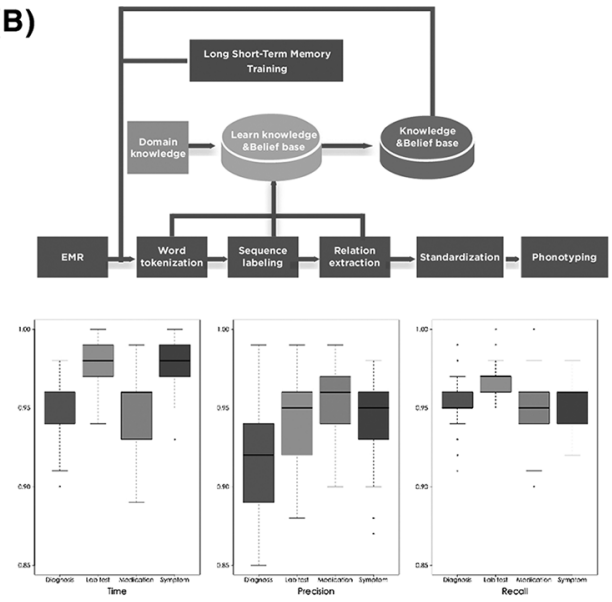

(D)

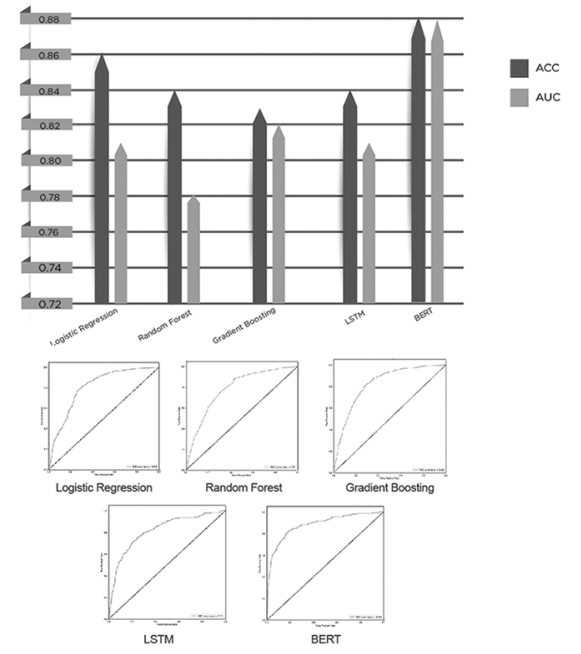

SLE (Systemic lupus Erythematosus) ～LSTM (Long Short-Term Memory) $\quad$ LN (lupus Nephritis) B BERT(Bidirectional Encoder Representations from Transformers) 